\title{
Sufficient Conditions for Decentralized \\ Potential Functions Based Controllers using \\ Canonical Vector Fields
}

\author{
Dimos V. Dimarogonas
}

\begin{abstract}
A combination of dual Lyapunov analysis and properties of decentralized navigation function based controllers is used to check the stability properties of a certain class of decentralized controllers for navigation and collision avoidance in multi-agent systems. The derived results yield a less conservative condition from previous approaches, which relates to the negativity of the sum of the minimum eigenvalues of the Hessian matrices at the critical points, instead of requiring each of the eigenvalues to be negative itself. This provides an improved characterization of the reachable set of this class of decentralized navigation function based controllers, which is less conservative than the previous results for the same class of controllers.
\end{abstract}

\section{INTRODUCTION}

Navigation of multi-agent systems is an area of increasing interest both from a research as well as an application viewpoint. When it comes to robots or vehicles, collision avoidance and decentralization are two important design specs for guaranteeing safety and scalability. Thus there has been a growing demand for the development of decentralized navigation methods with guaranteed collision avoidance. In recent years the application of potential field based methods has been explored [7],[18] as a promising alternative for such algorithms.

The author is with the ACCESS Linnaeus Center, School of Electrical Engineering, Royal Institute of Technology (KTH), Stockholm, Sweden dimosakth. se. He is also with the KTH Centre of Autonomous Systems. This work is supported by the Swedish Research Council (VR) through contract 2009-3948. A conference version of this work has been submitted to the 50th IEEE Conference on Decision and Control and European Control Conference. 
A common problem with potential field based path planning algorithms in multi-agent systems is the existence of local minima [10],[12]. The seminal work [11] involved navigation of a single robot in an environment of spherical obstacles with guaranteed convergence. In previous work, the closed-loop single robot navigation methodology of [11] was extended to multi-agent systems. In [13],[9],[16],[4], [7],[8] this method was extended to take into account the volume of each robot while formation control for point agents using navigation functions was dealt with in [21], [3]. Decentralized navigation functions were also used for multiple UAV guidance in [2].

Analysis of potential field based controllers via density functions was considered previously in [14] for centralized and in [6], [5] for decentralized multi-agent navigation. In this work we extend the previous results by combining the canonical vector field formulation of [14] with the dual Lyapunov analysis of decentralized potential fields in [5]. We examine the convergence of the system using a combination of primal and dual [19] Lyapunov techniques. This combination has been used in [15],[1],[14],[22]. In particular in [14],[15] a density function is provided for a single robot driven by a navigation function in a static obstacle workspace. Primal analysis is used to show convergence to a neighborhood of the critical points and density functions are used to prove the instability of the undesirable critical points using the properties of the navigation functions. The difference in our case is that we consider a system of multiple moving agents driven by decentralized potential functions and the potential functions are not considered $a$ priori navigation functions. On the contrary, the designed potentials are tuned properly to satisfy appropriate conditions to guarantee asymptotic stability from almost all initial conditions.

The significant outcome of the analysis is a less conservative sufficient condition for almost global navigation in the decentralized case. In [16],[4] the analysis relied on Morse theory that required, among others, that the minimum eigenvalues of the Hessian matrices for each decentralized navigation function at the critical points is strictly negative. In [6], [5] we derived a similar conclusion using density functions. Here we show that it is sufficient that only the sum of the minimum eigenvalues is strictly negative, and not the minimum eigenvalue of each decentralized navigation function itself. This yields an improved set of conditions for navigation.

More specifically, in [4],[7] the navigation functions were designed in such a way to allow agents that had already reached their destination to cooperate with the rest of the team in the case of a possible collision. In this paper, a construction similar to the initial navigation function construction in [11] is used. Hence each agent no longer participates in the collision avoidance 
procedure if its initial condition coincides with its desired goal. In essence, the agents might converge to critical points which are no longer guaranteed not to coincide with local minima. In [5] it was shown that in this case, agents converge to a sphere around their target points and an estimate of this was given. It turns out that with the formulation of this paper a less conservative bound can be derived that tends to zero in the case of point agents. Moreover, a broader set of initial conditions for navigation is derived for the case of non-point agents, establishing a direct correspondence between the convergence set radius and agents' maximum radii.

The rest of the paper is organized as follows: Section II presents the system and decentralized multi-agent navigation problem treated in this paper. The necessary mathematical preliminaries are provided in Section III, while Section IV provides the decentralized control design. Section $\mathrm{V}$ includes the convergence analysis and a simulated example is found in Section VI. Section VII summarizes the results of the paper and indicates further research directions.

\section{Definitions And Problem Statement}

Consider $N$ agents operating in a planar spherical workspace $W \subset \mathbb{R}^{2}$, with radius $R_{W}$. Let $q_{i} \in \mathbb{R}^{2}$ denote the position of agent $i$, and let $q=\left[q_{1}^{T}, \ldots, q_{N}^{T}\right]^{T}$ be the stack vector of all agents' positions. Denote $u=\left[u_{1}^{T}, \ldots, u_{N}^{T}\right]^{T}$. Agent motion is described by the single integrator:

$$
\dot{q}_{i}=u_{i}, i \in \mathcal{N}=\{1, \ldots, N\}
$$

where $u_{i}$ is the control input for each agent. We consider cyclic agents of specific radius $\varrho_{i} \geq$ $0, i \in \mathcal{N}$. Collision avoidance between the agents is meant in the sense that no intersections occur between the agents' discs. Each agent is assumed to have knowledge of the position of agents located in a cyclic neighborhood of specific radius $d$ at each time instant, where $d>\max _{i, j \in \mathcal{N}}\left(\varrho_{i}+\varrho_{j}\right)$. The function $\gamma_{d i}$ is agent's $i$ goal function which is minimized once the desired objective with respect to this particular agent is fulfilled. In particular, let $q_{d i} \in W$ denote the desired destination point of agent $i$. We then define $\gamma_{d i}=\left\|q_{i}-q_{d i}\right\|^{2}$ as the squared distance of the agent's $i$ configuration from $q_{d i}$.

In order to encode collisions, we define a function $\gamma_{i j}$, for $j=1, \ldots, N, j \neq i$, given by

$$
\gamma_{i j}\left(\beta_{i j}\right)=\left\{\begin{array}{l}
\frac{1}{2} \beta_{i j}, 0 \leq \beta_{i j} \leq c^{2} \\
\phi\left(\beta_{i j}\right), c^{2} \leq \beta_{i j} \leq d^{2}-\left(\varrho_{i}+\varrho_{j}\right)^{2} \\
1, d^{2}-\left(\varrho_{i}+\varrho_{j}\right)^{2} \leq \beta_{i j}
\end{array}\right.
$$


where $\beta_{i j}=\left\|q_{i}-q_{j}\right\|^{2}-\left(\varrho_{i}+\varrho_{j}\right)^{2}$. We also define the function $\gamma_{i 0}$ which refers to the workspace boundary (indexed by 0 ) and is used to maintain the agents within $W$. We have $\beta_{i 0}=\left(R_{W}-\varrho_{i}\right)^{2}-\left\|q_{i}\right\|^{2} ; \gamma_{i 0}$ is defined in the same way as $\gamma_{i j}, j>0$. The positive scalar $c$ and the function $\phi$ are chosen so that $\gamma_{i j}$ is everywhere twice continuously differentiable. For example, we can chose $\phi$ to be a fifth degree polynomial function whose coefficients are calculated so that $\gamma_{i j}$ is everywhere twice continuously differentiable. More details on the construction of $\gamma_{i j}$ and $\phi$ can be found in [5]. In the sequel, we will also use the notation $\nabla_{i}(\cdot) \triangleq \frac{\partial}{\partial q_{i}}(\cdot)$ for brevity.

\section{Mathematical Preliminaries}

\section{A. Dual Lyapunov Theory}

For functions $V: \mathbb{R}^{n} \rightarrow \mathbb{R}$ and $f: \mathbb{R}^{n} \rightarrow \mathbb{R}^{n}$ the notation

$$
\nabla V=\left[\begin{array}{lll}
\frac{\partial V}{\partial x_{1}} & \cdots & \frac{\partial V}{\partial x_{n}}
\end{array}\right]^{T}, \nabla \cdot f=\frac{\partial f_{1}}{\partial x_{1}}+\ldots+\frac{\partial f_{n}}{\partial x_{n}}
$$

is used. The dual Lyapunov result of [19] is stated as follows:

Theorem 1: Given the equation $\dot{x}(t)=f(x(t))$, where $f \in C^{1}\left(\mathbb{R}^{n}, \mathbb{R}^{n}\right)$ and $f(0)=0$, suppose there exists a nonnegative density function $\rho \in C^{1}\left(\mathbb{R}^{n} \backslash\{0\}, \mathbb{R}\right)$ such that $\rho(x) f(x) /\|x\|$ is integrable on $\left\{x \in \mathbb{R}^{n}:\|x\| \geq 1\right\}$ and

$$
[\nabla \cdot(f \rho)](x)>0 \text { for almost all } x
$$

Then, for almost all initial states $x(0)$ the trajectory $x(t)$ exists for $t \in[0, \infty)$ and tends to zero as $t \rightarrow \infty$. Moreover, if the equilibrium $x=0$ is stable, then the conclusion remains valid even if $\rho$ takes negative values.

Note that while Theorem 1 applies to the whole $\mathbb{R}^{n}$, we apply it here for the workspace $W$. The application of density functions to navigation function based systems was also used in [14]. A local version of Theorem 1 was used in [20]. Relaxed conditions for convergence to an equilibrium point in subsets of $\mathbb{R}^{n}$ were provided in [17].

\section{Decentralized Navigation Functions}

In [4],[7] the control law allowed agents that had already reached their desired destination to cooperate with the rest of the team in the case of a possible collision. In this paper, we use a construction similar to the initial navigation function construction in [11]. Hence each agent no 
longer participates in the collision avoidance procedure if its initial condition coincides with its destination. As a result, the derived decentralized potential functions are not guaranteed to avoid local minima, due to some of the agents already being located at their destinations. An analysis of the proposed potentials for point agents was held in [5] using dual Lyapunov theory [19]. Here we extend the above results to non-point agents and derive less conservative conditions for convergence by decentralizing the canonical vector fields framework introduced in [14].

Specifically, a decentralized potential function $\varphi_{i}: \mathbb{R}^{2 N} \rightarrow[0,1]$ is defined as

$$
\varphi_{i}=\frac{\gamma_{d i}}{\left(\gamma_{d i}^{k}+G_{i}\right)^{1 / k}}
$$

where $k>0$ is a scalar positive parameter, and the function $G_{i}$ is constructed in such a way in order to render the motion produced by the negated gradient of $\varphi_{i}$ with respect to $q_{i}$ repulsive with respect to the other agents. A proposed control law is of the form

$$
u_{i}=-K \nabla_{i} \varphi_{i}
$$

where $K>0$ is a positive scalar gain.

\section{A. Construction of the $G_{i}$ function}

In the sequel we review briefly the construction of $G_{i}$ for each agent $i$, which was introduced in [4], [7] for the case of local sensing capabilities. The multi-agent team is associated with an (undirected) graph whose vertices are indexed by the team members.

A binary relation with respect to an agent $i$ is an edge between agent $i$ and another agent. Binary relations represent collision schemes between pairs of agents. However, we need to distinguish between the cases of a collision scheme with one, two, or more agents. We use the term relation to describe all such possible collision schemes. A relation with respect to agent $i$ is defined as a set of binary relations with respect to agent $i$, which represents all pairs of agents that participate in a collision scheme with respect to $i$. The relation level is the number of binary relations in a relation with respect to agent $i$. The complementary set $\left(R_{j}^{i, C}\right)_{l}$ of relation $j$ with respect to agent $i$ is the set that contains all the relations of the same level apart from the specific relation $j$. The function $\gamma_{i j}$ is called the "Proximity Function" between agents $i$ and $j$ and serves as a metric for binary relations. Let $R_{k}^{i}$ denote the $k^{\text {th }}$ relation of level $l$ with respect to $i$. A metric for this relation is the "Relation Proximity Function" (RPF) which is defined as 
$\left(b_{R_{k}^{i}}\right)_{l}=\sum_{j \in\left(R_{k}^{i}\right)_{l}} \gamma_{i j}$ where $j \in\left(R_{k}^{i}\right)_{l}$ denotes the agents that participate in the relation. Thus an RPF is the sum of the Proximity Functions of the binary relations of the relation in hand. We also use the simplified notation $b_{r}^{i}=\sum_{j \in P_{r}} \gamma_{i j}$ for the RPF for simplicity, where $r$ denotes a relation and $P_{r}$ denotes the set of agents participating in the specific relation with respect to $i$.

We next introduce a function that distinguishes between the possible collision schemes. In particular, a "Relation Verification Function" (RVF) is defined by:

$$
\left(g_{R_{k}^{i}}\right)_{l}=\left(b_{R_{k}^{i}}\right)_{l}+\frac{\lambda\left(b_{R_{k}^{i}}\right)_{l}}{\left(b_{R_{k}^{i}}\right)_{l}+\left(B_{R_{k}^{i, C}}\right)_{l}^{1 / h}}
$$

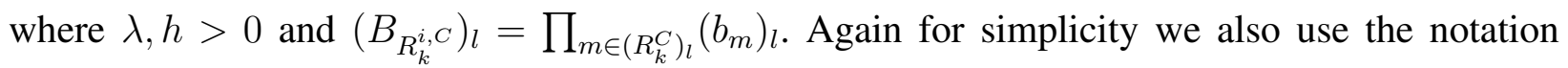
$\left(B_{R_{k}^{i, C}}\right)_{l} \equiv \tilde{b}_{r}^{i}=\prod_{\substack{s \in S_{r} \\ s \neq r}} b_{s}^{i}$ for the term $\left(B_{R_{k}^{i, C}}\right)_{l}$ where $S_{r}$ denotes the set of relations in the same level with relation $r$. The RVF is also written as $g_{r}^{i}=b_{r}^{i}+\frac{\lambda b_{r}^{i}}{b_{r}^{i}+\left(\tilde{b}_{r}^{i}\right)^{1 / h}}$. We have he following limits of RVF: (a) $\lim _{b_{r}^{i} \rightarrow 0} \lim _{\tilde{b}_{r}^{i} \rightarrow 0} g_{r}^{i}\left(b_{r}^{i}, \tilde{b}_{r}^{i}\right)=\lambda$ (b) $\lim _{\substack{b_{r}^{i} \rightarrow 0 \\ \tilde{b}_{r}^{i} \neq 0}} g_{r}^{i}\left(b_{r}^{i}, \tilde{b}_{r}^{i}\right)=0$. These limits guarantee that RVF will behave as an indicator of a specific relation. The function $G_{i}$ is defined as $G_{i}=$ $\prod_{l=1}^{n_{L}^{i}} \prod_{j=1}^{n_{R_{l}}^{i}}\left(g_{R_{j}^{i}}\right)_{l}$ where $n_{L}^{i}$ the number of levels and $n_{R_{l}}^{i}$ the number of relations in level- $l$ with respect to $i$. Hence $G_{i}$ is the product of the RVF's of all relations wrt $i$. Using the simplified notation, $G_{i}=\prod_{r=1}^{N_{i}} g_{r}^{i}$ where $N_{i}$ is the number of all relations with respect to $i$.

We then have $\nabla_{i} \varphi_{i}=\frac{\left(\gamma_{d i}^{k}+G_{i}\right)^{1 / k} \nabla_{i} \gamma_{d i}-\frac{\gamma_{d i}}{k}\left(\gamma_{d i}^{k}+G_{i}\right)^{1 / k-1}\left(k \gamma_{d i}^{k-1} \nabla_{i} \gamma_{d i}+\nabla_{i} G_{i}\right)}{\left(\gamma_{d i}^{k}+G_{i}\right)^{2 / k}}$, so that

$$
\nabla_{i} \varphi_{i}=\left(\gamma_{d i}^{k}+G_{i}\right)^{-1 / k-1}\left(G_{i} \nabla_{i} \gamma_{d i}-\frac{\gamma_{d i}}{k} \nabla_{i} G_{i}\right)
$$

We can also compute

$$
\nabla_{i} \varphi_{j}=\left(\gamma_{d j}^{k}+G_{j}\right)^{-1 / k-1}\left(-\frac{\gamma_{d j}}{k} \nabla_{i} G_{j}\right)
$$

A critical point of $\varphi_{i}$ is defined by $\nabla_{i} \varphi_{i}=0$. The following Proposition will be useful:

Proposition 1: For every $\epsilon>0$ there exists a positive scalar $P(\epsilon)>0$ such that if $k \geq P(\epsilon)$ then there are no critical points of $\varphi_{i}$ in the set $F_{i}=\left\{q \in W \mid g_{r}^{i} \geq \epsilon, \forall r=1, \ldots, N_{i}\right\} \backslash\left\{\gamma_{d i}=0\right\}$. Proof: At a critical point, we have $\nabla_{i} \varphi_{i}=0$, or $G_{i} \nabla_{i} \gamma_{d i}=\frac{\gamma_{d i}}{k} \nabla_{i} G_{i}$, which implies $2 k G_{i}=$ $\sqrt{\gamma_{d i}}\left\|\nabla_{i} G_{i}\right\|$, since $\left\|\nabla_{i} \gamma_{d i}\right\|=2 \sqrt{\gamma_{d i}}$. A sufficient condition for this equality not to hold in $F_{i}$ is given by $k>\frac{\sqrt{\gamma_{d i}}\left\|\nabla_{i} G_{i}\right\|}{2 G_{i}}, \forall q \in F_{i}$. An upper bound for the right hand side is given by $\frac{\sqrt{\gamma_{d i}}\left\|\nabla_{i} G_{i}\right\|}{2 G_{i}} \leq \frac{\sqrt{\gamma_{d i}}}{2} \sum_{r=1}^{N_{i}} \frac{\left\|\nabla_{i} g_{r}^{i}\right\|}{g_{r}^{i}} \leq \frac{1}{2 \varepsilon} \max _{W}\left\{\sqrt{\gamma_{d i}}\right\} \sum_{r=1}^{N_{i}} \max _{W}\left\{\left\|\nabla_{i} g_{r}^{i}\right\|\right\} \triangleq P$, since $g_{r}^{i} \geq \epsilon, \forall r=$ $1, \ldots, N_{i}$. Note that $\max _{W}\left\{\sqrt{\gamma_{d i}}\right\}, \max _{W}\left\{\left\|\nabla_{i} g_{r}^{i}\right\|\right\}$ are bounded due to the boundedness of $W . \diamond$ 


\section{CANONICAL VECTOR FiELDS FOR DNF's}

In this section we redefine the canonical vector fields' framework defined in [14] for centralized navigation functions in the decentralized case.

Let $q_{k i}$ be the $k$-th critical point for agent $i$, with $k=1, \ldots, n_{s i}$, where $n_{s i}$ the number of critical points. Similarly to [14], let $d_{k i}$ denote the distance between the agent position and the corresponding critical point, i.e., $d_{k i}=\left\|q_{i}-q_{k i}\right\|^{2}$. Let $\lambda_{\min _{i}}\left(q_{k i}\right)$ be the minimum eigenvalue of the Hessian matrix $\frac{\partial^{2} \varphi_{i}}{\partial q_{i}^{2}}$ at $q_{i}=q_{k i}$ and $u_{k i}$ be the corresponding unit eigenvector. Define $U_{k i}=u_{k i} u_{k i}^{T}+\varepsilon_{1} I$ where $I$ is the two-dimensional unit matrix and $0<\varepsilon_{1} \leq 1$. Denote also $U_{n_{s i}+1, i}=U_{n_{s i}+2, i}=I$ and $d_{n_{s i}+1, i}=\varphi_{i}, d_{n_{s i}+2, i}=1-\varphi_{i}$. Define $\bar{d}_{k i}=\prod_{l=1, l \neq k}^{n_{s i}+2} d_{l i}$. Then for each agent $i$ we define the matrix $D_{\varphi_{i}}$ as $D_{\varphi_{i}}=\mu \sum_{k=1}^{n_{s i}+2} \frac{\bar{d}_{k i}}{d_{k i}+d_{k i}} U_{k i}$. It can be shown that $D_{\varphi_{i}}$ fulfills similar properties to the matrix $D_{\varphi}$ defined for a centralized navigation function in [14]. In particular, the following result holds:

Lemma 2: The matrix $D_{\varphi_{i}}$ has the following properties: (i) $D_{\varphi_{i}}=\mu U_{k i}$, for $q_{i}=q_{k i}$ (ii) $D_{\varphi_{i}}=\mu I$, for $G_{i}=0$ (iii) $D_{\varphi_{i}}=\mu I$, for $q_{i}=q_{d i}$, (iv) $\nabla_{i} D_{\varphi_{i}}=0$, for $q_{i}=q_{d i}$ and $\nabla_{i} D_{\varphi_{i}}=0$, for $q_{i}=q_{k i}$, and (v) $0<x^{T} D_{\varphi_{i}} x \leq 2\left(n_{s i}+2\right) \mu\|x\|^{2}$, for all $x \in \mathbb{R}^{2}$.

The last property guarantees positive definiteness and boundedness of $D_{\varphi_{i}}$.

We consider the modification of the control law (5) by using $D_{\varphi_{i}}$ as an additional gain matrix:

$$
u_{i}=-K D_{\varphi_{i}} \nabla_{i} \varphi_{i}
$$

System (9) is called the canonical system. Note that the two systems share the same critical points. Moreover, using the exact same arguments as in the proof of Proposition 3 in [14], the existence of an appropriate tuning of $\mu$ such that the trajectories of (5) are bounded by the trajectories of (9) can be established. This allows us to derive conclusions on the convergence of (5) by examining the convergence of (9).

\section{Convergence Analysis}

The convergence analysis of the overall system consists of two parts. The first part uses primal Lyapunov analysis to show that the system converges to an arbitrarily small neighborhood of the critical points. We then use dual Lyapunov analysis to show that the set of initial conditions that drives the system to points other than the goal configurations is of zero measure. 


\section{A. Primal Lyapunov Analysis}

The stability of system (1) under the control law (5) was analyzed in [5] and convergence to an arbitrarilly small neighborhood of the critical points was established. We now show that, as expected, the multiplication of the control with the positive definite matrix $D_{\varphi_{i}}$ yields the same behavior. Note that the closed loop kinematics of system (1) under (9) are given by

$$
\dot{q}=f(q)=\left[\begin{array}{l}
-K D_{\varphi_{1}} \nabla_{1} \varphi_{1} \\
\vdots \\
-K D_{\varphi_{N}} \nabla_{N} \varphi_{N}
\end{array}\right]
$$

Define $\varphi=\sum_{i} \varphi_{i}$. The derivative of $\varphi$ can be computed by

$$
\dot{\varphi}=(\nabla \varphi)^{T} \dot{q}=-K \sum_{i=1}^{N}\left(\nabla_{i} \varphi\right)^{T}\left(D_{\varphi_{i}} \nabla_{i} \varphi_{i}\right)=-K \sum_{i=1}^{N} \sum_{j=1}^{N}\left(D_{\varphi_{i}} \nabla_{i} \varphi_{i}\right)^{T}\left(\nabla_{i} \varphi_{j}\right)
$$

where $\varphi_{i}$ is defined in (4). Consider $\varepsilon>0$. Then we can further compute

$$
\begin{aligned}
& \dot{\varphi}=-K \sum_{i=1}^{N}\left(\left(\nabla_{i} \varphi_{i}\right)^{T} D_{\varphi_{i}} \nabla_{i} \varphi_{i}+\sum_{j \neq i}\left(\nabla_{i} \varphi_{j}\right)^{T}\left(D_{\varphi_{i}} \nabla_{i} \varphi_{i}\right)\right) \\
& =-K \sum_{i:\left\|\nabla_{i} \varphi_{i}\right\|>\varepsilon}\left(\left(\nabla_{i} \varphi_{i}\right)^{T} D_{\varphi_{i}} \nabla_{i} \varphi_{i}+\sum_{j \neq i}\left(\nabla_{i} \varphi_{j}\right)^{T}\left(D_{\varphi_{i}} \nabla_{i} \varphi_{i}\right)\right) \\
& -K \sum_{i:\left\|\nabla_{i} \varphi_{i}\right\| \leq \varepsilon}\left(\left(\nabla_{i} \varphi_{i}\right)^{T} D_{\varphi_{i}} \nabla_{i} \varphi_{i}+\sum_{j \neq i}\left(\nabla_{i} \varphi_{j}\right)^{T}\left(D_{\varphi_{i}} \nabla_{i} \varphi_{i}\right)\right) \\
& \leq-K \sum_{i:\left\|\nabla_{i} \varphi_{i}\right\|>\varepsilon}\left(\lambda_{\min }\left(D_{\varphi_{i}}\right) \varepsilon^{2}+\sum_{j \neq i}\left(\nabla_{i} \varphi_{j}\right)^{T}\left(D_{\varphi_{i}} \nabla_{i} \varphi_{i}\right)\right) \\
& -K \sum_{i:\left\|\nabla_{i} \varphi_{i}\right\| \leq \varepsilon}\left(\nabla_{i} \varphi_{j}\right)^{T}\left(D_{\varphi_{i}} \nabla_{i} \varphi_{i}\right)
\end{aligned}
$$

The terms in the first sum, where $\left\|\nabla_{i} \varphi_{i}\right\|>\varepsilon$, are lower bounded as follows: $\lambda_{\min }\left(D_{\varphi_{i}}\right) \varepsilon^{2}+$ $\sum_{j \neq i}\left(\nabla_{i} \varphi_{j}\right)^{T}\left(D_{\varphi_{i}} \nabla_{i} \varphi_{i}\right) \geq \lambda_{\min }\left(D_{\varphi_{i}}\right) \varepsilon^{2}-\varepsilon\left\|D_{\varphi_{i}}\right\|_{\max } \sum_{j \neq i}\left\|\nabla_{i} \varphi_{j}\right\|$. Using (8) we have $\left\|\nabla_{i} \varphi_{j}\right\|=$ $\left(\gamma_{d j}^{k}+G_{j}\right)^{-1 / k-1}\left(\frac{\gamma_{d j}}{k}\left\|\nabla_{i} G_{j}\right\|\right)$. For $\gamma_{d j}>\gamma_{\min }, k>1$, the term $\left(\gamma_{d j}^{k}+G_{j}\right)^{1 / k+1}$ is minimized by $\gamma_{\min }^{2}$ so that $\lambda_{\min }\left(D_{\varphi_{i}}\right) \varepsilon^{2}+\sum_{j \neq i}\left(\nabla_{i} \varphi_{j}\right)^{T}\left(D_{\varphi_{i}} \nabla_{i} \varphi_{i}\right) \geq \lambda_{\min }\left(D_{\varphi_{i}}\right) \varepsilon^{2}-\varepsilon \frac{\left\|D_{\varphi_{i}}\right\|_{\max }}{k \gamma_{\min }^{2}} \sum_{j \neq i} \gamma_{d j}\left\|\nabla_{i} G_{j}\right\|$. We want to achieve a bound of the form $\lambda_{\min }\left(D_{\varphi_{i}}\right) \varepsilon^{2}+\sum_{j \neq i}\left(\nabla_{i} \varphi_{j}\right)^{T}\left(D_{\varphi_{i}} \nabla_{i} \varphi_{i}\right) \geq \rho_{1}>0$, where $0<\rho_{1}<\lambda_{\min }\left(D_{\varphi_{i}}\right) \varepsilon^{2}$. A sufficient condition for this to hold is $\frac{(N-1)\left\|D_{\varphi_{i}}\right\|_{\max }}{k \gamma_{\min }^{2}} \max _{j \neq i}\left\{\gamma_{d j}\left\|\nabla_{i} G_{j}\right\|\right\} \leq$ $\frac{\lambda_{\min }\left(D_{\varphi_{i}}\right) \varepsilon^{2}-\rho_{1}}{\varepsilon}$, or equivalently

$$
k \geq \frac{\varepsilon}{\lambda_{\min }\left(D_{\varphi_{i}}\right) \varepsilon^{2}-\rho_{1}} \frac{(N-1)\left\|D_{\varphi_{i}}\right\|_{\max }}{\gamma_{\min }^{2}} \max _{j \neq i}\left\{\gamma_{d j}\left\|\nabla_{i} G_{j}\right\|\right\}
$$


We next compute a lower bound on the terms in the second sum, where $\left\|\nabla_{i} \varphi_{i}\right\| \leq \varepsilon$. Note that

$$
\begin{aligned}
\left(\nabla_{i} \varphi_{j}\right)^{T}\left(D_{\varphi_{i}} \nabla_{i} \varphi_{i}\right) & =\left(D_{\varphi_{i}} \nabla_{i} \varphi_{i}\right)^{T}\left(\nabla_{i} \varphi_{j}\right)=\frac{\left(G_{i} \nabla_{i} \gamma_{d i}-\frac{\gamma_{d i}}{k} \nabla_{i} G_{i}\right)^{T} D_{\varphi_{i}}^{T}\left(-\frac{\gamma_{d j}}{k} \nabla_{i} G_{j}\right)}{\left(\gamma_{d i}^{k}+G_{i}\right)^{1 / k+1}\left(\gamma_{d j}^{k}+G_{j}\right)^{1 / k+1}} \\
& =\frac{-\frac{\gamma_{d j} G_{i}}{k} \nabla_{i} \gamma_{d i}^{T} D_{\varphi_{i}}^{T} \nabla_{i} G_{j}+\frac{\gamma_{d j} \gamma_{d i}}{k^{2}} \nabla_{i} G_{i}^{T} D_{\varphi_{i}}^{T} \nabla_{i} G_{j}}{\left(\gamma_{d i}^{k}+G_{i}\right)^{1 / k+1}\left(\gamma_{d j}^{k}+G_{j}\right)^{1 / k+1}}
\end{aligned}
$$

so that $\left(\nabla_{i} \varphi_{j}\right)^{T}\left(D_{\varphi_{i}} \nabla_{i} \varphi_{i}\right) \geq \frac{1}{\gamma_{\min }^{4}}\left(-\frac{\gamma_{d j} G_{i}}{k}\left\|\nabla_{i} \gamma_{d i}\right\|\left\|D_{\varphi_{i}}||\right\| \nabla_{i} G_{j}\left\|-\frac{\gamma_{d j} \gamma_{d i}}{k^{2}}\right\| \nabla_{i} G_{i}\left\||| D_{\varphi_{i}}||\right\| \nabla_{i} G_{j} \|\right)$.

We want to achieve a bound of the form $\sum_{j \neq i}\left(\nabla_{i} \varphi_{j}\right)^{T}\left(D_{\varphi_{i}} \nabla_{i} \varphi_{i}\right) \geq-2 \rho_{2}$, where $\rho_{2}>$ 0. A sufficient condition for this to hold is that $\frac{1}{\gamma_{\min }^{4}} \frac{\gamma_{d j} G_{i}}{k}\left\|\nabla_{i} \gamma_{d i}\right\|\left\|D_{\varphi_{i}} \mid\right\| \nabla_{i} G_{j} \| \leq \rho_{2}$ and $\frac{1}{\gamma_{\min }^{4}} \frac{\gamma_{d j} \gamma_{d i}}{k^{2}}\left\|\nabla_{i} G_{i}\right\|\left\|D_{\varphi_{i}}||\right\| \nabla_{i} G_{j} \| \leq \rho_{2}$ or equivalently, that both

$$
k \geq \frac{\max _{j \neq i}\left\{\gamma_{d j} G_{i}\left\|\nabla_{i} \gamma_{d i}\right\|\left\|D_{\varphi_{i}} \mid\right\|\left\|\nabla_{i} G_{j}\right\|\right\}}{\rho_{2} \gamma_{\min }^{4}}
$$

and

$$
k \geq \sqrt{\frac{\max _{j \neq i}\left\{\gamma_{d i} \gamma_{d j}\left\|\nabla_{i} G_{i}\right\|\left\|D_{\varphi_{i}}\right\|\left\|\nabla_{i} G_{j}\right\|\right\}}{\rho_{2} \gamma_{\text {min }}^{4}}}
$$

hold. Provided that $k$ satisfies (10),(11),(12), we have $\dot{\varphi} \leq-K \rho_{1}+K(N-1)^{2} \rho_{2}$, assuming that there exists at least one agent such that $\left\|\nabla_{i} \varphi_{i}\right\|>\varepsilon$. The latter is strictly negative for $0<(N-1)^{2} \rho_{2}<\rho_{1}<\lambda_{\min }\left(D_{\varphi_{i}}\right) \varepsilon$.

In essence, $\dot{\varphi}$ can be rendered strictly negative as long as there exists at least one agent with $\left\|\nabla_{i} \varphi_{i}\right\|>\varepsilon$. Thus the system converges to an arbitrarily small region of the critical points, provided that $0<(N-1)^{2} \rho_{2}<\rho_{1}<\lambda_{\min }\left(D_{\varphi_{i}}\right) \varepsilon$ and the conditions on $k$ hold. We have:

Proposition 3: Consider the system (1) with the control law (9). Assume that $\gamma_{d i} \geq \gamma_{\min }>$ 0. Pick $\varepsilon>0, \rho_{1}, \rho_{2}>0$ satisfying $0<(N-1)^{2} \rho_{2}<\rho_{1}<\lambda_{\min }\left(D_{\varphi_{i}}\right) \varepsilon$ and assume that (10),(11),(12) hold. Then the system converges to the set $\left\|\nabla_{i} \varphi_{i}\right\| \leq \varepsilon$ for all $i$ in finite time.

We also refer to the corresponding convergence result for the system (5) in [5], Prop. 2.

\section{B. Dual Lyapunov Analysis}

Having established convergence to an arbitrarily small neighborhood of the critical points, density functions are now used to pose sufficient conditions that the attractors of undesirable critical points are sets of measure zero.

For $\varphi=\sum_{i} \varphi_{i}$, define $\rho=\varphi^{-\alpha}, \alpha>0$ which is defined for all points in $W$ other than the desired equilibrium $\gamma_{d i}=0$, for all $i \in \mathcal{N}$. Note also that each $\varphi_{i}$ is $C^{2}$ and takes values in $[0,1]$ 
and thus both the function $\varphi$ and its gradients are bounded functions in $W$. Hence, $\rho$ fulfils the integrability condition of Theorem 1 and is a suitable density function for the equilibrium point $\gamma_{d i}=0, \forall i \in \mathcal{N}$.

We have $\nabla \rho=-\alpha \varphi^{-\alpha-1} \nabla \varphi$ and $\nabla \cdot(f \rho)=\nabla \rho \cdot f+\rho \nabla \cdot f=-\alpha \varphi^{-\alpha-1} \nabla \varphi \cdot f+$ $\varphi^{-\alpha} \nabla \cdot f$. Whenever $\nabla_{i} \varphi_{i}=0$ for all $i \in \mathcal{N}$, we have $f=0$. Moreover, $\nabla \cdot f=\nabla$. $\left[-D_{\varphi_{1}} \nabla_{1} \varphi_{1}, \ldots,-D_{\varphi_{N}} \nabla_{N} \varphi_{N}\right]$. For $\nabla_{i} \varphi_{i}=0$ for all $i \in \mathcal{N}$, we can calculate $\nabla \cdot(f \rho)=$ $\varphi^{-\alpha} \nabla \cdot f=-\varphi^{-\alpha} \sum_{i}\left\{\mu \lambda_{\min _{i}}+\varepsilon_{1} \mu\left(\lambda_{\min _{i}}+\lambda_{\max _{i}}\right)\right\}$ where $\lambda_{\min _{i}}, \lambda_{\max _{i}}$ denote the minimum and maximum eigenvalue, respectively, of the Hessian matrix $\frac{\partial^{2} \varphi_{i}}{\partial q_{i}^{2}}$ at the particular critical point of agent $i$. The following result is then straightforward:

Proposition 4: Assume that $\sum_{i} \lambda_{\min _{i}}<0$. Then the right hand side of the last equation is rendered strictly positive by choosing

$$
\varepsilon_{1}<\frac{\left|\sum_{i} \lambda_{\min _{i}}\right|}{\left|\sum_{i}\left\{\lambda_{\min _{i}}+\lambda_{\max _{i}}\right\}\right|}
$$

The above result implies that a sufficient condition for the fulfillment of the condition $\nabla$. $(f \rho)>0$ for $\nabla_{i} \varphi_{i}=0$ for all $i \in \mathcal{N}$ is given by

$$
\sum_{i} \lambda_{\min _{i}}<0
$$

It thus turns out that negativity of the minimum Hessian eigenvalue of all $\varphi_{i}$ is a sufficient but not a necessary condition for decentralized navigation. This condition was used in [4] using tools from Morse Theory. Using the combination of dual Lyapunov functions and canonical vector fields, we derived the sufficient condition (14), which is less conservative than the Morse condition $\lambda_{\min _{i}}<0$ for all $i$. Moreover, the condition (14) is also less conservative than the condition $\sum_{i} \lambda_{\min _{i}}+\lambda_{\max _{i}}<0$ that was derived in our previous work [5] using the same tools as in the current paper, apart from the canonical vector field formulation.

Let us now elaborate a little more on the condition (14). Using the notation $H_{i}\left(\varphi_{i}\right) \triangleq \frac{\partial^{2} \varphi_{i}}{\partial q_{i}^{2}}$ for the Hessian matrix of $\varphi_{i}$, it is true that $\sum_{i} \lambda_{\min _{i}} \leq \sum_{i} \hat{u}_{i}^{T} H_{i} \hat{u}_{i}$ holds for all vectors $\hat{u}_{i}$ with $\left\|\hat{u}_{i}\right\|=1$. Note also that the critical points of $\varphi_{i}$ and $\hat{\varphi}_{i}=\frac{\gamma_{i}}{G_{i}}$ coincide [11],[4]. So (14) is implied by the existence of a vector $\hat{u}_{i}$ with $\left\|\hat{u}_{i}\right\|=1$ such that

$$
\sum_{i} \hat{u}_{i}^{T} H_{i}\left(\hat{\varphi}_{i}\right) \hat{u}_{i}<0
$$


Note that the corresponding sufficient condition based on the Morse property in [4] had the form $\hat{u}_{i}^{T} H_{i}\left(\hat{\varphi}_{i}\right) \hat{u}_{i}<0$ for all $i \in \mathcal{N}$. From Proposition 1 we know that at a critical point of $\hat{\varphi}_{i}$, we have $g_{r}^{i} \leq \epsilon$ for at least one relation of agent $i$. With a slight abuse of notation, we will denote $g_{r}^{i}=g_{i}$ in the sequel for brevity. Consider now $\hat{u} \triangleq\left\{\frac{\nabla_{1} b_{1}\left(q_{c}\right)^{\perp}}{\left\|\nabla_{1} b_{1}\left(q_{c}\right)^{\perp}\right\|}, \ldots, \frac{\nabla_{N} b_{N}\left(q_{c}\right)^{\perp}}{\left\|\nabla_{N} b_{N}\left(q_{c}\right)^{\perp}\right\|}\right\}$ and $\hat{u}_{i} \triangleq \frac{\nabla_{i} b_{i}\left(q_{c}\right)^{\perp}}{\left\|\nabla_{i} b_{i}\left(q_{c}\right)^{\perp}\right\|}$, where $q_{c} \in C_{\hat{\varphi}_{i}}$, and $C_{\hat{\varphi}_{i}}$ is the set of critical points of $\hat{\varphi}_{i}$. By its definition $\hat{u}_{i}$ is orthogonal to $\nabla_{i} b_{i}$ at a critical point $q_{c}$, and so $\hat{u}_{i}^{T} \cdot \nabla_{i} b_{i}=0$ and $\nabla_{i} b_{i}^{T} \cdot \hat{u}_{i}=0$.

We can now use similar calculations to the ones used in the proof of Lemma 5 in [4] to derive the following expression:

$$
\sum_{i} \hat{u}_{i}^{T} H_{i}\left(\hat{\varphi}_{i}\right) \hat{u}_{i}=\sum_{i} \frac{\gamma_{d i}^{k-1}}{G_{i}^{2}}\left\{\bar{g}_{i} c_{i} \mu_{i}+g_{i}\left(\gamma_{d i} \eta_{i}-\gamma_{d i} \xi_{i}+\frac{\nabla_{i} \bar{g}_{i}^{T} \nabla_{i} \gamma_{d i}}{2}-\sigma_{i}\right)\right\}
$$

where $\bar{g}_{i}=\prod_{l=1, l \neq r}^{N_{i}} g_{l}^{i}, \mu_{i}=\frac{1}{2} \nabla_{i} b_{i}^{T} \nabla_{i} \gamma_{d i}-v_{i} \gamma_{d i}, v_{i}=2\left|P_{r}\right|>2, c_{i}=1+\frac{\lambda}{b_{i}+\tilde{b}_{i}^{1 / h}}, \xi_{i}=\hat{u}_{i}^{T}$. $\nabla_{i}^{2} \bar{g}_{i} \hat{u}_{i}+\frac{\bar{g}_{i}}{c_{i}} \cdot \hat{u}_{i}^{T} A_{i} \hat{u}_{i}-2 \frac{\lambda}{c_{i}\left(b_{i}+\tilde{b}_{i}^{1 / h}\right)^{2}} \hat{u}_{i}^{T} \nabla_{i} \tilde{b}_{i}^{1 / h} \nabla_{i} \bar{g}_{i} \hat{u}_{i}$

$$
\begin{gathered}
\eta_{i}=\left(1-\frac{1}{k}\right)\left[\begin{array}{l}
\frac{\hat{u}_{i}^{T} \nabla_{i} \bar{g}_{i} \nabla_{i} \bar{g}_{i}^{T} \hat{u}_{i}}{\bar{g}_{i}}-2 \lambda \frac{\hat{u}_{i}^{T} \nabla_{i} \bar{g}_{i}\left(\nabla_{i} \tilde{b}_{i}^{1 / h}\right)^{T} \hat{u}_{i}}{c_{i}\left(b_{i}+\tilde{b}_{i}^{1 / h}\right)^{2}} \\
+\lambda^{2} \bar{g}_{i} \frac{\hat{u}_{i}^{T} \nabla_{i} \tilde{b}_{i}^{1 / h}\left(\nabla_{i} \tilde{b}_{i}^{1 / h}\right)^{T} \hat{u}_{i}}{c_{i}^{2}\left(b_{i}+\tilde{b}_{i}^{1 / h}\right)^{4}}
\end{array}\right], \\
\sigma_{i}=\frac{\lambda \bar{g}_{i}}{2 c_{i}\left(b_{i}+\tilde{b}_{i}^{1 / h}\right)^{2}}\left(\nabla_{i} b_{i}+\nabla_{i} \tilde{b}_{i}^{1 / h}\right)^{T} \nabla_{i} \gamma_{d i},
\end{gathered}
$$

and $A_{i}=\lambda\left[\begin{array}{l}2 \frac{\left(\nabla_{i} b_{i}+\nabla_{i} \tilde{b}_{i}^{1 / h}\right)\left(\nabla_{i} b_{i}+\nabla_{i} \tilde{b}_{i}^{1 / h}\right)^{T}}{\left(b_{i}+\tilde{b}_{i}^{1 / h}\right)^{3}}- \\ -\frac{\left(\nabla_{i}^{2} b_{i}+\nabla_{i}^{2} \tilde{b}_{i}^{1 / h}\right)}{\left(b_{i}+\tilde{b}_{i}^{1 / h}\right)^{2}}\end{array}\right]$.

Note that the second term in the parenthesis in (16) can be made arbitrarily small by a small choice of $\epsilon$ but can still be positive, so the first term should be strictly negative. In particular, the condition

$$
\sum_{i} \frac{\gamma_{d i}^{k-1}}{G_{i}^{2}} \bar{g}_{i} c_{i}\left(\frac{1}{2} \nabla_{i} b_{i}^{T} \nabla_{i} \gamma_{d i}-v_{i} \gamma_{d i}\right)<0
$$

is a sufficient condition for (15) to hold. Note that $v_{i}>2$. Moreover, for $0 \leq g_{i} \leq \epsilon$ we have $0 \leq b_{i}=\sum_{j \in P_{r}} \beta_{i j} \leq \epsilon$ and thus $0 \leq \beta_{i j} \leq \epsilon$ for all $j \in P_{r}$, for the particular relation $r$ with respect to agent $i$. We then have $\left\|\nabla_{i} b_{i}\right\|=\left\|2 \sum_{j \in P_{r}}\left(q_{i}-q_{j}\right)\right\| \leq 2 \sum_{j \in P_{r}}\left\|\left(q_{i}-q_{j}\right)\right\| \leq 2 \sum_{j \in P_{r}} \sqrt{\epsilon+\left(\varrho_{i}+\varrho_{j}\right)^{2}}$. Moreover $\left\|\nabla_{i} \gamma_{d i}\right\|=2 \sqrt{\gamma_{d i}}$ and we shall use the notation $M_{i}=\frac{\gamma_{d i}^{k-1}}{G_{i}^{2}} \bar{g}_{i} c_{i} \sqrt{\gamma_{d i}}$ in the sequel. 
Denote also by $\bar{\gamma}=\bar{\gamma}(q)=\min _{i} \gamma_{d i}(q)$ the minimum of the functions $\gamma_{d i}$ at each point $q$ of the workspace. It can be seen that the sufficient condition (17) is now implied by

$$
\sum_{i} M_{i} \sum_{j \in P_{r}} \sqrt{\epsilon+\left(\varrho_{i}+\varrho_{j}\right)^{2}}<\sum_{i} M_{i} \sqrt{\bar{\gamma}}
$$

which is in turn implied by

$$
\max _{i}\left\{\sum_{j \in P_{r}} \sqrt{\epsilon+\left(\varrho_{i}+\varrho_{j}\right)^{2}}\right\}<\sqrt{\bar{\gamma}}
$$

For the case of common equal radii $\varrho$ for all agents, the above is simplified to $\max _{i}\left\{\sum_{j \in P_{r}} \sqrt{\epsilon+4 \varrho^{2}}\right\}<$ $\sqrt{\bar{\gamma}}$. Since the maximum number of binary relations in a relation can be equal to 6 in the case of decentralized navigation functions, the above is implied by

$$
\bar{\gamma}>36\left(\epsilon+4 \varrho^{2}\right)
$$

We now use the argument of [14] mentioning that since (3) is satisfied exactly at the critical points, it is satisfied also in an arbitrary small neighborhood around them. From the primal Lyapunov analysis, we know that indeed the system converges to an arbitrarily small neighborhood of the critical points. The dual Lyapunov analysis guarantees that the attractors of the undesirable critical points are sets of measure zero. The following then holds:

Proposition 5: Consider (1) with the control law (9). Let the assumptions of Propositions 1,3 hold. Pick arbitrarily small $\epsilon, \gamma_{\min }$ such that $\epsilon \geq \gamma_{\min }>0$. Then for almost all initial conditions the closed loop system (1), (9) (i) fulfills (3) as long as $\sqrt{\bar{\gamma}}>\max _{i}\left\{\sum_{j \in P_{r}} \sqrt{\epsilon+\left(\varrho_{i}+\varrho_{j}\right)^{2}}\right\}$ for the case of agents with non equal radii and $\bar{\gamma}>36\left(\epsilon+4 \varrho^{2}\right)$ for the case of agents with equal radii and (ii) converges to the set $\sqrt{\bar{\gamma}} \leq \max _{i}\left\{\sum_{j \in P_{r}} \sqrt{\epsilon+\left(\varrho_{i}+\varrho_{j}\right)^{2}}\right\}$ for the case of agents with non equal radii, and to the set $\bar{\gamma} \leq 36\left(\epsilon+4 \varrho^{2}\right)$ for the case of agents with equal radii.

The latter along with the fact that the trajectories of (5) are bounded by the trajectories of (9) for appropriate tuning of $\mu$, guarantees that the above Proposition holds also for the closed loop system (1), (5). Statement (i) in the above proposition can be rephrased as: provided that the agents are located at configurations satisfying (20) or (19), they are guaranteed to navigate towards their destinations. Thus as long (20) or (19) are satisfied, the closed loop system navigates towards the final destinations, as these are depicted by statement (ii). The optimal outcome would be the case that all agents converged (almost) simultaneously to the sets described by $\sqrt{\gamma_{d i}} \leq \max _{i}\left\{\sum_{j \in P_{r}} \sqrt{\epsilon+\left(\varrho_{i}+\varrho_{j}\right)^{2}}\right\}$ for the case of agents with non equal radii, 
and to the set $\gamma_{d i} \leq 36\left(\epsilon+4 \varrho^{2}\right)$ for the case of agents with equal radii. This relates to the issue of synchronization in this framework, which motivates an interesting future research direction.

Note that for point agents, (20) becomes

$$
\bar{\gamma}>36 \epsilon
$$

This establishes that for point agents, as long as $\bar{\gamma}>36 \epsilon$ holds, agents navigate towards their destinations. Since $\epsilon$ can be chosen arbitrarily small, convergence to an arbitrarily small neighborhood around the desired destination points is guaranteed for the case of point agents.

\section{Simulations}

The derived results are now supported through a computer simulation.

Note that the analysis yields an improved minimum on $\bar{\gamma}$ with respect to [6] since it relies on the fact that only the sum of the minimum eigenvalues of the Hessians at critical points needs to be negative, and not every minimum eigenvalue itself. The analysis in [6] yields a minimum of $\bar{\gamma}$ which is of the order $\frac{R_{W}^{2}}{\epsilon}$. Thus the results of [6] are significantly more conservative than the current paper, which yields a $\bar{\gamma}$ of order $\epsilon+4 \varrho^{2}$, since in general $\varrho<<R_{W}$. This point is depicted in the simulation scenario, where we consider four agents whose initial positions do not satisfy the sufficient condition for $\bar{\gamma}$ given in [6] while they satisfy (19). In particular, the initial and final destinations of the four agents are given by $q_{1}(0)=[-0.2,-0.2]^{T}, q_{2}(0)=[-0.2,0.21]^{T}, q_{3}(0)=$ $[0.21,-0.2]^{T}, q_{4}(0)=[0.2,0.2]^{T}$ and $q_{d 1}=[0.21,0.2]^{T}, q_{d 2}=[0.2,-0.2]^{T}, q_{d 3}=[-0.2,0.2]^{T}$, $q_{d 4}=[-0.2,-0.2]^{T}$ respectively. The control parameters are given by $k=100, \varrho_{1}=\ldots=$ $\varrho_{4}=\varrho=0.05, d=0.13, R_{w}=1$. It is easily verified that the system satisfies (19) at the initial positions while it does not satisfy the conditions of [6].

Since agents satisfy (19), the results of Proposition 5 are applicable. Plots I-IV of Fig. 1 show the evolution of the closed-loop system. In plot I, the initial position and desired destination of agent $i, i=1,2,3,4$ are denoted by $A i, T i$ respectively. Each agent is represented by a disc. The arrows indicate the agent motion at each plot. In plot II, the agents follow an almost straight line towards their destination, until they get into a conflict situation which is resolved in plot III. In plot IV, the agents have reached their desired destinations. 

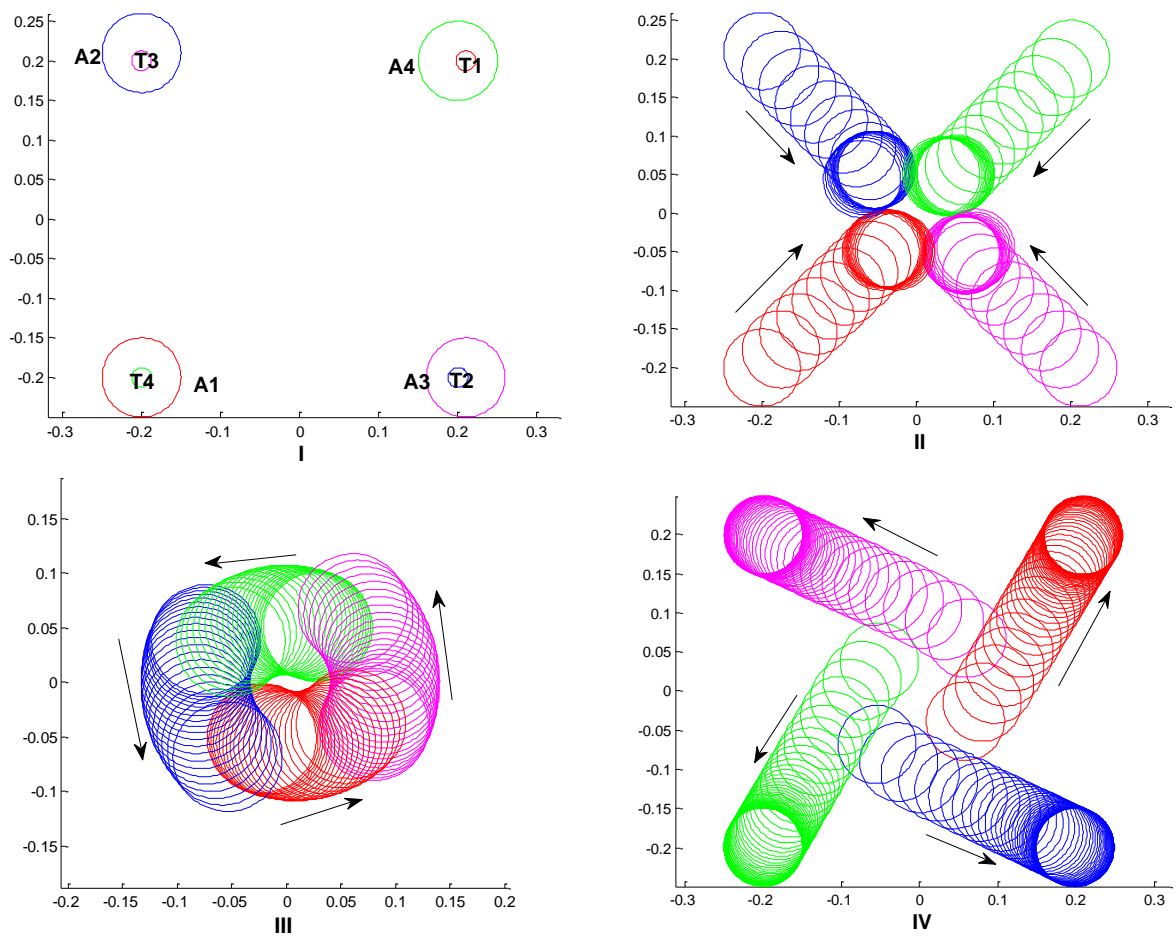

Fig. 1. Four agents initially satisfying (19) converge to their destinations. The agents do not fulfill the conditions of [6].

\section{CONCLUSIONS}

A combination of dual Lyapunov analysis and properties of decentralized navigation function based controllers were used to check the stability properties of a class of decentralized controllers for navigation and collision avoidance in multi-agent systems. The derived results yield a less conservative condition which relates to the negativity of the sum of the minimum eigenvalues of the Hessian matrices at the critical points, instead of requiring each of the eigenvalue to be negative itself. This provides an improved characterization of the reachable set of this certain class of decentralized navigation function based controllers, which is less conservative than the previous results for the same class of controllers.

\section{REFERENCES}

[1] D. Angeli. An almost global notion of input-to-state stability. IEEE Transactions on Automatic Control, 49(6):866-874, 2004.

[2] J. Chen, D.M. Dawson, M. Salah, and T. Burg. Multiple UAV navigation with finite sensing zone. 2006 American Control Conference, pages 4933-4938. 
[3] M.C. DeGennaro and A. Jadbabaie. Formation control for a cooperative multiagent system with a decentralized navigation function. 2006 American Control Conference, pages 1346-1351.

[4] D. V. Dimarogonas, S. G. Loizou, K.J. Kyriakopoulos, and M. M. Zavlanos. A feedback stabilization and collision avoidance scheme for multiple independent non-point agents. Automatica, 42(2):229-243, 2006.

[5] D.V. Dimarogonas and E. Frazzoli. Analysis of decentralized potential field based multi-agent navigation via primal-dual lyapunov theory. 49th IEEE Conf. Decision and Control, pages 1215-1220, 2010.

[6] D.V. Dimarogonas and K.J. Kyriakopoulos. An application of Rantzer's dual Lyapunov theorem to decentralized navigation. 15th IEEE Mediterranean Conference on Control and Automation, 2007.

[7] D.V. Dimarogonas and K.J. Kyriakopoulos. Decentralized navigation functions for multiple agents with limited sensing capabilities. Journal of Intelligent and Robotic Systems, 48(3):411-433, 2007.

[8] A. Ghaffarkhah and Y. Mostofi. Communication-aware target tracking using navigation functions - centralized case. International Conference on Robot Communication and Coordination (RoboComm), 2009.

[9] C.S. Karagoz, H.I. Bozma, and D.E. Koditschek. Coordinated navigation of multiple independent disk-shaped robots. Tech. report no. ms-cis-07-16, Department of Computer and Information Science, University of Pennsylvania, 2007.

[10] O. Khatib. Real-time obstacle avoidance for manipulators and mobile robots. International Journal of Robotics Research, 5(1):90-98, 1986.

[11] D. E. Koditschek and E. Rimon. Robot navigation functions on manifolds with boundary. Advances Appl. Math., 11:412442, 1990.

[12] S. LaValle. Planning Algorithms. Cambridge University Press, 2007.

[13] S. G. Loizou and K. J. Kyriakopoulos. Closed loop navigation for multiple holonomic vehicles. Proc. of IEEE/RSJ Int. Conf. on Intelligent Robots and Systems, pages 2861-2866, 2002.

[14] S.G. Loizou and A. Jadbabaie. Density functions for navigation function based systems. IEEE Transactions on Automatic Control, 53(2):612-617, 2008.

[15] S.G. Loizou and V. Kumar. Weak input-to-state stability properties for navigation function based controllers. 45th IEEE Conf. Decision and Control, pages 6506-6511, 2006.

[16] S.G. Loizou and K.J Kyriakopoulos. Navigation of multiple kinematically constrained robots. IEEE Transactions on Robotics, 24(1):221-231, 2008.

[17] I. Masubuchi. Analysis of positive invariance and almost regional attraction via density functions with converse results. IEEE Transactions on Automatic Control, 52(7):1329-1333, 2007.

[18] L. Pallottino, V.G. Scordio, A. Bicchi, and E. Frazzoli. Decentralized cooperative policy for conflict resolution in multivehicle systems. IEEE Transactions on Robotics, 23(6):1170-1183, 2007.

[19] A. Rantzer. A dual to Lyapunov's stability theorem. Systems and Control Letters, 42:161-168, 2001.

[20] A. Rantzer and S. Prajna. On analysis and synthesis of safe control laws. 42nd Allerton Conference on Communication, Control, and Computing, 2004.

[21] H.G. Tanner and A. Kumar. Formation stabilization of multiple agents using decentralized navigation functions. Robotics: Science and Systems, 2005.

[22] J.F. Vasconcelos, A. Rantzer, C. Silvestre, and P. Oliveira. Combination of lyapunov functions and density functions for stability of rotational motion. 48th IEEE Conf. Decision and Control, pages 5941-5946, 2009. 